yan.tex

\title{
Instability and Periodic Deformation in Bilayer Membranes Induced by Freezing
}

\author{
Yan Jie ${ }^{1}$, Zhou Haijun ${ }^{1}$, Ou-Yang Zhong-can ${ }^{1,2}$ \\ ${ }^{1}$ Institute of Theoretical Physics, Chinese Academy of Sciences, \\ P.O. Box 2735, Beijing 100080, China \\ ${ }^{2}$ Center for Advanced Study, Tsinghua University, Beijing 100084, China
}

(January 17, 2014)

\begin{abstract}
The instability and periodic deformation of bilayer membranes during freezing processes are studied as a function of the difference of the shape energy between the high and the low temperature membrane states. It is shown that there exists a threshold stability condition, bellow which a planar configuration will be deformed. Among the deformed shapes, the periodic curved square textures are shown being one kind of the solutions of the associated shape equation. In consistency with recent experimental observations, the optimal ratio of period and amplitude for such a texture is found to be approximately equal to $\sqrt{2} \pi$.
\end{abstract}

PACS numbers:87. 22. Bt, 82. 70. -y, 82. 65. -i 
Lipid molecules, such as phospholipids, assemble into bilayer membranes (BMs) in aqueous environment. On the elasticity and statistics of closed BMs (vesicles), many investigations have been taken on[1-4]. At high enough concentration of lipids the BMs will convert from vesicles into extended configurations[5]. For extended membranes, experiment demonstrated the existence of one-dimensional periodic cylindrical surfaces[6]. It is found theoretically that such kind of shapes can be well explained by the Helfrich curvature elasticity theory for $\mathrm{BMs}[7]$. Recent experiments employing freeze fracture electron microscopy method revealed the existence of stable periodic curved square textures (PCSTs), which resemble egg cartons and look equal from both sides of the membrane[8-10]. Later on, Klösgen and Helfrich found a grainy texture in vesicular bilayers of egg yolk phosphatidylcholine with cryo-transmission electron microscopy[11]. In fact, periodic deformations of cell membrane are abundant in nature[12], and a common aspect of these observations[8-12] is that all these curved textures are created through rapid cooling processes.

On the theoretical side, Meyer conjected the observed periodic shape to be infinite periodic minimal surface (IPMS)[13]. As shown in Ref. [14], the formation of IPMS requires the regular arrangement of proteins or other globular macromolecules, however, this condition is not necessary for the formation of the PCSTs reported in Refs. [8] and [9]. Recently, an approach for the problem is proposed by Goetz and Helfrich by suggesting a new curvature elasticity model in which bending energy terms higher than quadratic order in the principal curvatures are included and used to Monte Carlo simulation(MCS) for yielding PCSTs[15]. They found that if the MCS is carried out initially from a plane, then the plane remains unchanged apart from its fluctuations, which indicates the existence of an energy barrier between a planar and any periodicly curved shapes[15]. As an analytic check for the problem, our first task in this Letter is to show that if taking account only curvature elastic energy (no matter whether higher order energy terms be included or not), a plane will always be a stable configuration and an energy barrier must be overcome if some possible stable curved pattern is to be realized. Therefore, the question on the origin of the formation of PCSTs is still open. As the second step, the main propose in this Letter, the PCST formation is considered as the result of the quench of the temperature decreasing. We analytically derived the equilibrium-shape equation for the BMs by taking account of competition among the curvature elasticity, the volume Gibbs free energy difference between the high and the low temperature membrane states, and the surface tension energy difference. The sum of these three energies can be understood as the shape formation energy (see bellow for details). The planar configuration is a trivial solution of the shape equation, and for small violation from a plane, a PCST described by triangular functions is also another (approximate) solution if the differences of Gibbs free energy density and tension density satisfy some threshold condition. We show that below such a threshold condition the shape formation energy of a planar $\mathrm{BM}$ in the quench process could become negative. In other words, the planar BM becomes unstable in the freezing process, as a result, curved BMs will be formed spontaneously to keep the equilibrium condition (i.e. the zero shape formation energy). Taking into consideration of the equilibrium condition in the quench-like cooling processes, the above argument provides an insight for the mechanism of the PCST deformation for the BMs. The optimal ratio of the periods and amplitudes of the PCSTs formed in such processes is shown to be approximately equal to $\sqrt{2} \pi$. This result is well consistent with recent observations of the PCST formation in BMs by several groups[8-10]. 
First, we check whether the instability of a planar configuration is caused by higher order energy terms or not. We follow the work of Goetz and Helfrich[15] and express the bending energy as

$$
F_{B}=\int\left[\frac{1}{2} \kappa H^{2}+\bar{\kappa} K+\kappa_{2} K^{2}+\kappa_{4} K^{4}\right] d A,
$$

where $H=\left(c_{1}+c_{2}\right) / 2$ and $K=c_{1} c_{2}$ are, respectively, the mean curvature and the Gaussian curvature of the membrane surface, $\kappa$ is the bending rigidity and $\bar{\kappa}$ is the modulus of Gaussian curvature, $d A$ is the surface area element, $\kappa_{2}$ is supposed to be negative[15]. Considering periodic deformations of the flat membrane, we omit the $\bar{\kappa}$ term because of the Gauss-Bonnet theorem. The general form of a surface in the Cartesian coordinate system is described as

$$
\mathbf{Y}(x, y)=(x, y, z(x, y))
$$

The corresponding equilibrium-shape equation for the surface described by Eq. (2) is obtained by requiring the first order variation of its elastic energy (1) with small deformations, $\delta^{(1)} F_{B}$, equal to zero. This condition is satisfied if $[16,17]$

$$
\kappa H^{3}-\kappa H K+2 \kappa_{2} H K^{2}+6 \kappa_{4} H K^{4}+\frac{1}{2} \kappa \nabla^{2} H+2 \kappa_{2} \bar{\nabla}^{2} K+4 \kappa_{4} \bar{\nabla}^{2} K^{3}=0,
$$

where $\nabla^{2}=(1 / \sqrt{g}) \partial_{i}\left(\sqrt{g} g^{i j} \partial_{j}\right)$ is the Laplace-Beltrami operator, and $\bar{\nabla}^{2}$ is a new operator defined as $(1 / \sqrt{g}) \partial_{i}\left(\sqrt{g} K L^{i j} \partial_{j}\right)$, here $\partial_{1}=\partial_{x}, \partial_{2}=\partial_{y}, g=\operatorname{det}\left(g_{i j}\right),\left(L^{i j}\right)=\left(L_{i j}\right)^{-1}$, and $g_{i j}$, $L_{i j}$ are associated with the first and second fundamental forms of the surface, respectively $[16,17]$. Eq. (3) is a highly nonlinear differential equation and generally it is very difficult to find exact solutions. The egg carton surfaces obtained by Goetz and Helfrich[15] may be considered as a numerical periodic solution of Eq. (3). Although the planar configuration, such as $\mathbf{Y}=(x, y, 0)$ with its $H=K=0$, is a exact solution of it, the second variation of $F_{B}$ for the plane has been calculated as

$$
\delta^{(2)} F_{B}=\frac{\kappa}{8} \int\left(\partial_{x x} z+\partial_{y y} z\right)^{2} d x d y
$$

where $z(x, y)$ denotes small deviation of the plane along the normal direction $(0,0,1)$. Obviously, Eq. (4) is positive for any violations of a plane since $\kappa$ is always positive. Note that $\delta^{(2)} F_{B}$ is irrelevant to $\kappa_{2}$ and $\kappa_{4}$, which indicates the stability of the plane is irrelevant to the elastic energy terms higher than quadratic order in the principal curvatures. To demonstrate this point more generally, we also analyzed the following curvature elasticity model which includes the complete terms up to the fourth order in the principle curvatures

$$
F_{B}=\int\left[\kappa H^{2}+\bar{\kappa} K+\kappa_{3} H^{3}+\bar{\kappa}_{3} H K+\kappa_{4} H^{4}+\bar{\kappa}_{4} H^{2} K+\kappa_{4}^{*} K^{2}\right] d A
$$

and found the plane is still a strict solution of the corresponding shape equation. However, the second variation of the energy at the plane is found to be the same as Eq. (4), i.e., irrelevant with higher order energy terms. The second variation $\delta^{(2)} F_{B}>0$ indicates the existence of an energy barrier between the flat configuration and any possible stable curved ones, which is in accordance with the result of Goetz and Helfrich[15] in their simulations. 
The deformation of a plane to a PCST by freezing as observed in Ref. [8-10] thus can not be explained by considering only curvature elastic energy. Considering the above mentioned difficulty, in the following we will investigate this problem from another viewpoint, in which the temperature decreasing plays a key role in the instability of the planar configuration.

Generally, when the temperature of the BM is decreased from a high value $\left(T_{h}\right)$ to a low value $\left(T_{l}\right)$, the values of the volume Gibbs free energy density and the surface tension will change correspondingly because lipid molecules will arrange themselves more orderly in the membrane. Thus, the conversion of the membrane from a high temperature state to a low temperature state during the quench-like process is quite similar to the tube formation of a smectic-A phase grown from isotropic phase in liquid crystal[16, 18] and the coil formation of a multishell carbon nanotubes grown from the carbonaceous mesophase[19]. As shown in Refs. [16, 18, 19], if take the high temperature state of the membranes as the zero energy state, the shape formation energy at the low temperature is the sum of the following three terms, (i) the net difference of the volume Gibbs free energy between the $T_{l}$ and $T_{h}$ states, i.e., $F_{V}=-g_{0} V=-g_{0} d_{0} A$ where $V, A$, and $d_{0}$ are the volume, area, and thickness of the BM, respectively, and $g_{0}$ (of positive value) is the difference of the volume Gibbs free energy densities between the $T_{h}$ and $T_{l}$ states, (ii) the surface tension energy difference $F_{A}=2\left(\gamma\left(T_{l}\right)-\gamma\left(T_{h}\right)\right) A$ where $\gamma(T)$ is the surface tension at temperature $T$, and (iii) the Helfrich curvature elastic energy $F_{B}$. For symmetric BMs, $F_{B}=(\kappa / 2) \int H^{2} d A+\bar{\kappa} \int K d A[1]$, (in the following we will also omit the $\bar{\kappa}$ term).Here we do not take account of higher order elastic terms, because this simplest curvature elastic model is enough for following calculations. The total shape formation energy is then

$$
\begin{aligned}
F=F_{V}+F_{A}+F_{B} & =-g_{0} d_{0} \int d A+2\left(\gamma\left(T_{l}\right)-\gamma\left(T_{h}\right)\right) \int d A+\frac{\kappa}{2} \int H^{2} d A \\
& =\lambda \int d A+\frac{\kappa}{2} \int H^{2} d A,
\end{aligned}
$$

where $\lambda=-g_{0} d_{0}+2\left(\gamma\left(T_{l}\right)-\gamma\left(T_{h}\right)\right)$.

The variational equation of $\delta F=0$ yields the equilibrium-shape equations of the BM[16, $17]$

$$
\kappa H^{3}-\kappa H K+\frac{\kappa}{2} \nabla^{2} H-2 \lambda H=0
$$

It is obvious that a planar BM is always a solution of the shape equation (7), and the corresponding shape formation energy of the planar $\mathrm{BM}$ is $F=\lambda A$. The shape formation energy is regarded as a free energy and the equilibrium threshold condition of $F=0$ yields the criteria for the stability of a planar BM with the change of temperature from $T_{h}$ to $T_{l}$ as

$$
\lambda=-g_{0} d_{0}+2\left(\gamma\left(T_{l}\right)-\gamma\left(T_{h}\right)\right)=0
$$

This equation describes the threshold relationship between $\gamma$ and $g_{0}$ for a planar BM. We emphasize that both $\gamma$ and $g_{0}$ are dependent on the temperatures and environment conditions. The deformation procedure for BMs, which is a sudden cooling, is actually a sort of quench-like process. As long as the shape formation energy for the planar BMs deviates downwards from the threshold condition, i.e., $\lambda$ becomes negative, the resultant remnant part of energy will prevent the planar BM from keeping stable. Then a shape deformation 
will be induced and it would lead to another solution of the shape equation with its shape formation energy again being equal to zero. In fact, $g_{0}$ will increase with the temperature decreasing; moreover, it is argued in Ref. [20] that when temperature decreases, hydrocarbon chains of lipid molecules will become less flexible and correspondingly the thickness $d_{0}$ will increase slightly. Considering these two effects, according to Eq. (8), $\lambda$ may become negative and the planar BM may be curved under the (quick) cooling process. These features give a natural explanation for the deformation of BMs.

Generally, to find exact periodic solutions to Eq. (7) is quite difficult. However, to find a threshold deformation from planar solution to the PCST observed in experiments[8-10] we need only to consider a small deformation away from a planar configuration and try to find some approximate periodic solutions to Eq. (7). The deformed surface is described by Eq. (2). Keeping up to the first order in $z(x, y)$, Eq. (7) reduces to

$$
\kappa \Delta H-4 \lambda H=0
$$

with

$$
H=(1 / 2) \Delta z,
$$

where $\Delta=\partial_{x x}+\partial_{y y}$ is the usual two dimensional Laplace operator. We seek PCST solutions to Eq. (9), which becomes possible as long as the value of $\lambda$ changes from positive to negative values. One such solution is

$$
\mathbf{Y}(x, y)=\left(x, y, z_{a}\left[\cos \left(2 \pi\left(x-x_{0}\right) / p\right)+\cos \left(2 \pi\left(y-y_{0}\right) / p\right)\right]\right),
$$

where $x_{0}$ and $y_{0}$ are integration constants, and $p$ is the period, $p=2 \pi \sqrt{\kappa /(-4 \lambda)}, z_{a}$ is the amplitude of the deformation. A schematic representation of the form of this configuration is shown in Fig. 1, the resemblance of Fig. 1 with experimental observations[8-10] is obvious. Based on this agreement, we have confidence to believe that the configuration represented by Eq. (11) does reveal some essential properties of PCSTs. In the following we will discuss some details.

The metric of this PCST surface is

$$
\begin{aligned}
\sqrt{g}= & \sqrt{1+\partial_{x}^{2} z+\partial_{y}^{2} z}=\sqrt{1+4 \pi^{2} z_{a}^{2} / p^{2}}\left(1-\frac{2 \pi^{2} z_{a}^{2}\left[\cos \left(2 p\left(x-x_{0}\right)\right)+\cos \left(2 p\left(y-y_{0}\right)\right)\right]}{p^{2}+4 \pi^{2} z_{a}^{2}}\right)^{1 / 2} \\
& \approx \sqrt{1+4 \pi^{2} z_{a}^{2} / p^{2}} .
\end{aligned}
$$

The total surface area of the BM is regarded as conserved, so Eq. (12) indicates that the total projected area $A_{p}$ of this PCST on the $x y$ plane is less than the total area $A$ of the undeformed planar configuration, $A_{p} / A=1 / \sqrt{1+4 \pi^{2} z_{a}^{2} / p^{2}}=p / \sqrt{p^{2}+4 \pi^{2} z_{a}^{2}}$. Considering Eqs. (10-12) and the conservation of total surface area, the formation energy of this periodic $\mathrm{BM}$ can be derived from Eq. (6) as

$$
F=\lambda A\left(1-\frac{2 \pi^{2} z_{a}^{2}}{p^{2}}\right)
$$

Since now we have the periodic curved situation which is different from the planar BM case, we may treat the threshold condition $F=0$ as 


$$
\frac{p}{z_{a}}=\sqrt{2} \pi
$$

Eq. (14) gives the optimal ratio of the period and the amplitude for the PCSTs. Under this condition, the ratio $A_{p} / A=1 / \sqrt{3} \approx 0.6$, i.e., the area projection on the $x y$ plane will cover only about 60 percent of the total area occupied by a undeformed planar surface. Of course, when the amplitude $z_{a}$ becomes comparable with the period, (as is the case in Eq. (14)), the surface described by Eq. (11) can no longer satisfy the equilibrium-shape equation (7), so it is just a rough approximation of actual stable PCSTs observed in experiments. Even though, we find that the relationship of Eq. (14) holds well for actual systems, indicating the validity of the proposed mechanism for PCST formation. As shown in Fig. 3b of Ref. [9], there is a fraction of BMs being regularly curved and the amplitude of this deformed texture can be estimated from the boundary curve of this region. By a direct evaluation from this figure, we do find $p / z_{a} \approx \sqrt{2} \pi$ hold well. A rough estimation from the periodic deformed part of this figure gives $p \approx 25 \mathrm{~nm}$ and $z_{a} \approx 5 \mathrm{~nm}$, i.e., $p / z_{a}=5 \approx \sqrt{2} \pi$. Another square texture, shown in Fig. 4 d of Ref. [8], gives $p / z_{a} \approx 3 / 0.5=6$, again close to the prediction of Eq. (14).

It is also interesting to study the value of $g_{0}$ for the observed PCSTs of the recent experiments[8-10]. We set $d_{0}=4 \mathrm{~nm}[15,21], \kappa=4 \times 10^{-19} \mathrm{~J}[20]$. As for the surface tension, according to de Gennes and Taupin[22], $\gamma=2 \kappa /\left(R R_{0}\right)$ where $R_{0}$ is the spontaneous radius of curvature and $R$ is the actual radius of curvature, so $\gamma\left(T_{h}\right)=0[22]$ for the planar shape at $T_{h}$, because $R=\infty$, and for the PCST shape at $T_{l}$, as an estimation we can set $R \approx R_{0} \approx p$, hence $\gamma\left(T_{l}\right) \approx 2 \kappa / p^{2}$. Then for a typical PCST with period $p=45 \mathrm{~nm}$ to occur, $g_{0}$ should equal to about $6 \times 10^{5} \mathrm{~J} / \mathrm{m}^{3}$. This value is consistent with the experimental conclusion of Sternberg et al [9] that the formation of PCSTs is not connected with temperature-induced lipid phase transition processes. For if some phase transition should occur, some thermal energy at least about $1.5-2 \mathrm{kcal} / \mathrm{mol}$ would be released at the transition point alone, according to Ref. [21]. For a typical BM where each lipid molecule occupies an average volume of about $1000 A^{3}$ [21], it is equivalent to say that there is at least a thermal energy difference about $1 \times 10^{7} \mathrm{~J} / \mathrm{m}^{3}$ exists between the high and the low temperature states, if a phase transition does occur. However, the value we estimated from experiments, about $6 \times 10^{5} \mathrm{~J} / \mathrm{m}^{3}$, is much smaller than this value. So it is shown that there is no phase transition processes in the PCST formation with temperature decreasing, which is in agreement with the observation of Ref. [9].

In summary, by deriving the expression for the formation energy of the BMs as well as the equilibrium-shape equation, we have shown that there exists a threshold condition for a planar BM to be stable, below which the planar BMs become unstable and will undergo a shape deformation. In particular, we have shown the existence of periodic deformations and given an estimate of the optimal ratio of the period and the amplitude, i.e., $p / z_{a} \approx \sqrt{2} \pi$, which is consistent with the recent experiment observations[8-10].

This work is partly supported by the National Natural Science Foundation of China. 


\section{REFERENCES}

[1] P. B. Canham, J. Theor. Biol. 26, 61 (1970); W. Helfrich, Z. Naturforsch. 28C, 693 (1973).

[2] For recent reviews, see U. Seifert, Adv. Phys. 46, 13 (1997); U. Seifert and R. Lipowsky, in Handbook of Biological Physics, edited by R. Lipowsky and E. Sackmann (NorthHolland, 1995), Vol. 1A, pp. 403.

[3] H. Deuling and W. Helfrich, J. Phys. (Paris) 37, 1335 (1976); J. Jenkins, J. Math. Biophys. 4, 149 (1977); M. Peterson, J. Appl. Phys. 57, 1739 (1985); S. Svetina and B. Zeks, Euro. Biophys. J. 17, 101 (1989); L. Miao, B. Fourcade, M. Rao, M. Wortis, and R. Zia, Phys. Rev. A43, 6843 (1991); L. Miao, B.Fourcade, M. WOrtis, and H. Döbereiner, Phys. Rev. E49, 5389 (1994).

[4] U. Seifert, Phys. Rev. Lett. 66, 2404 (1991); U. Seifert, K. Berndl, and R. Lipowsky, Phys. Rev. A44, 1182 (1991); J. Berndl, J. Käs, R. Lipowsky, E. Sackmann, and U. Seifert, Europhys. Lett. 13, 659 (1990).

[5] J. N. Israelachvili, Intermolecular and surface forces (Academic, New York, 1991).

[6] W.Harbich and W. Helfrich, Chem. Phys. Lipids 36, 39 (1984).

[7] Zhang Shao-guang and Ou-Yang Zhong-can, Phys. Rev. E53, 4206 (1996); Zhang Shaoguang, Acta Physica Sinica (Overseas Ed.), 6, 641 (1997).

[8] H. W. Meyer, W. Richter and J. Gumpert, Biochim. Biophys. Acta 1026, 171 (1990).

[9] B. Sternberg, J. Gumpert, G. Reinhardt and K. Gawrisch, ibid. 898, 223 (1987).

[10] J. F. Tocanne, P. H. Jh. Vorrergaert, A. T. Verkleij, and L. L. M. van Deenen, Chem. Phys. Lipids 12, 220 (1974).

[11] B. Klösgen and W. Helfrich, Eur. Biophys. J. 22, 329 (1993).

[12] D. W. Fawcett, The Cell (W. B. Saunders, Philadelphia, 1981), 2nd ed.

[13] H. W. Meyer, Acta Biotechnol. 10, 135 (1990).

[14] S. T. Hyde and S. Andersson, Z. Kristallogr. 174, 237 (1986); S. Andersson, S. T. Hyde, K. Larsson and S. Lidlin, Chem. Rev. 88, 221 (1988).

[15] R. Goetz and W. Helfrich, J. Phys. II France 6, 215 (1996).

[16] H. Naito, M. Okuda, and Ou-Yang Zhong-can, phys. Rev. Lett. 70, 2912 (1993); Phys. Rev. E52, 2095 (1995).

[17] Ou-Yang Zhong-can and W. Helfrich, Phys. Rev. A39, 5280 (1989).

[18] H. Naito, M. Okuda, and Ou-Yang Zhong-can, Phys. Rev. E55, 1655 (1997).

[19] Ou-Yang Zhong-can, Z.-B. Su, and C.-L. Wang, Phys. Rev. Lett. 78, 4055 (1997).

[20] S. Komura, in Vesicles, edited by M. Posoff (Marcel Dekker, New York, 1996), Chapter VI.

[21] J. N. Israelachvili, S. Marčelja and R. G. Horn, Q. Rev. Biophys. 13, 121 (1980).

[22] P. G. de Gennes and C. Taupin, J. Phys. Chem. 86, 2294 (1982). 


\section{FIGURES}

FIG. 1. Schematic representation of the periodic curved square texture solution. The ratio of period and amplitude is set equal to $\sqrt{2} \pi$ (see text for details). 


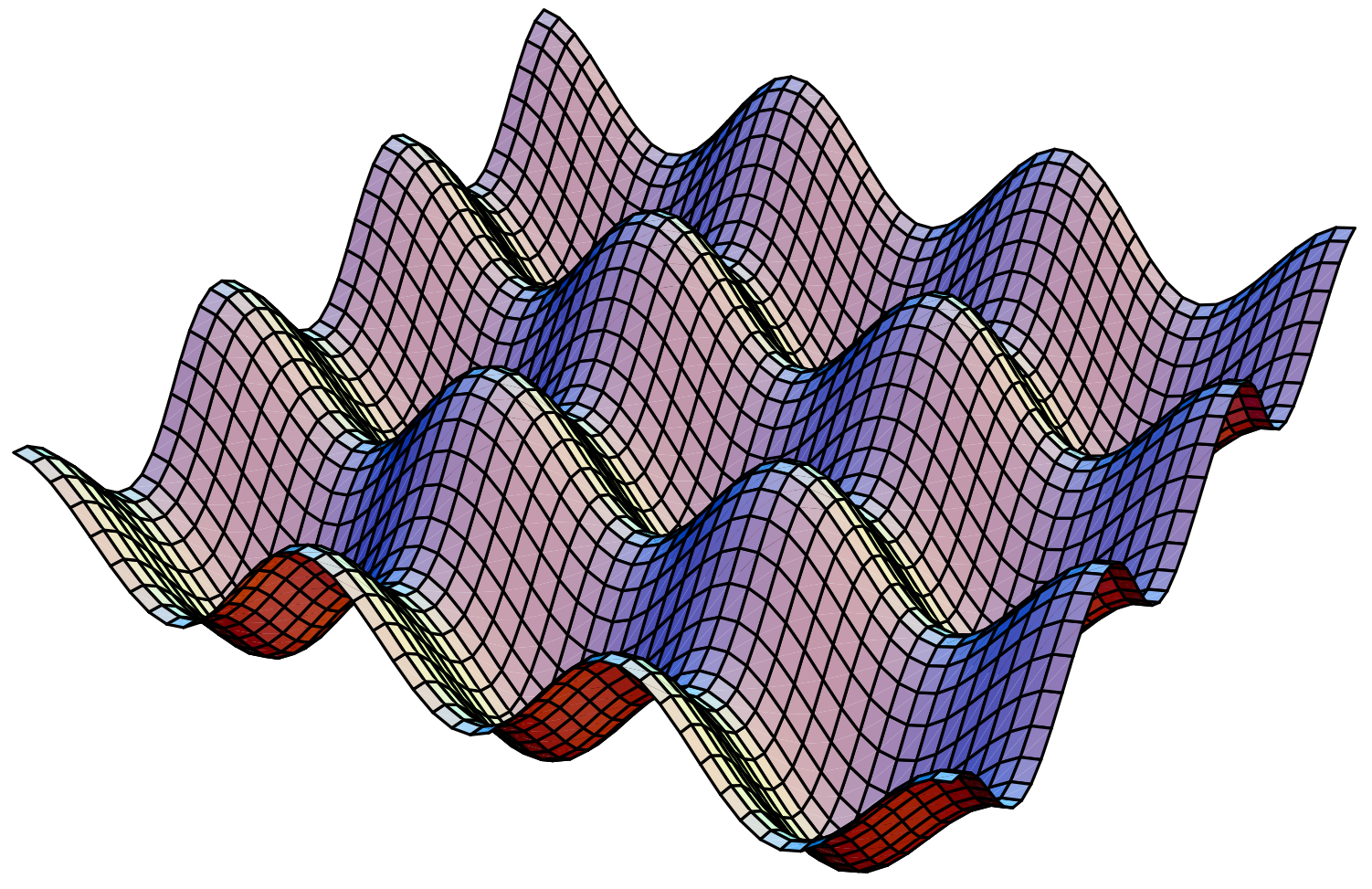

